\title{
ISOLATION THEOREM FOR PRODUCTS OF LINEAR FORMS
}

\author{
T. W. CUSICK
}

\begin{abstract}
A theorem of Cassels and Swinnerton-Dyer about products of three linear forms with real coefficients is generalized to products of any number of linear forms.
\end{abstract}

1. Introduction. In this paper we generalize some results of Cassels and Swinnerton-Dyer [3]. Suppose $f\left(x_{1}, \ldots, x_{n}\right)$ is a product of $n \geq 3$ linear forms with real coefficients. Our first theorem is an isolation theorem for these forms $f\left(x_{1}, \ldots, x_{n}\right)$. For a discussion of the significance of isolation theorems in the geometry of numbers see Cassels [2, pp. 264-265]; for examples and applications of isolation theorems see [2, pp. 286-298].

In order to state our theorem we need to define an $\varepsilon$-neighborhood of the form $f$. For any $\varepsilon>0$, an $\varepsilon$-neighborhood of $f$ is the set of all products of $n$ linear forms such that the coefficients of the linear forms are within $\varepsilon$ of the corresponding coefficients of the linear forms in $f$. Any set which contains some $\varepsilon$-neighborhood of $f$ will be called a neighborhood of $f$.

THEOREM 1. Let $f\left(x_{1}, \ldots, x_{n}\right)$ be the product of $n \geq 3$ linear forms with real coefficients. Suppose that $f$ has integer coefficients and that $f=0$ only when all the $x_{i}$ are 0 . Let $\left(\delta_{1}, \delta_{2}\right)$ be any open interval. Then there is a neighborhood of $f$ such that all forms in the neighborhood which are not multiples of $f$ itself take some value in the interval $\left(\delta_{1}, \delta_{2}\right)$ for some integer values of the variables $x_{1}, \ldots, x_{n}$.

It is well known (see [2, pp. 285-286]) that the conditions imposed on the product $f$ of linear forms in Theorem 1 imply that $f$ is equal to an integer times the product of all the $n$ conjugates of one linear form whose coefficients are algebraic integers in some totally real algebraic number field of degree $n$. Thus Theorem 1 is really a statement about norm forms. Of course any norm form $f$ satisfies $\inf |f|>0$, where the infimum is taken over all integers $x_{1}, \ldots, x_{n}$ not all zero. It is a notorious unsolved problem [2, pp. 260-264] to decide whether norm forms are the only products of $n \geq 3$ linear forms with this property. For $n=2$, this problem has a negative answer because we can find a binary quadratic form

$$
f(x, y)=\left(x+\theta_{1} y\right)\left(x+\theta_{2} y\right)=x^{2}+\beta x y+\gamma y^{2}
$$

with $\gamma$ irrational such that inf $|f(x, y)| \geq 1$ for $x, y$ not both zero. Such forms exist as long as $\beta^{2}-4 \gamma \geq 9$, and indeed there are uncountably many with $\beta^{2}-4 \gamma=9$ (see Cassels [1, Lemma 14, pp. 38-39]). Also, the analog of Theorem 1 is false for $n=2$, for instance if we consider the form $x^{2}-3 y^{2}$. A certain weaker isolation theorem [2, pp. 287-289] is valid for $n=2$.

Received by the editors September 26, 1985 and, in revised form, March 25, 1986. 1980 Mathematics Subject Classification (1985 Revision). Primary 10E15; Secondary 10C10, 10 F99. Key words and phrases. Linear forms, norm forms. 
The case $n=3$ of Theorem 1 was proved by Cassels and Swinnerton-Dyer [3, Theorem 2, p. 74]. Skubenko [4, 5] also claims a proof of Theorem 1, but his argument is difficult to follow (in particular, when he uses Lemma 3, Corollary in [4] to prove the theorem of that paper, he needs to show, in his notation, that $H^{2} \varepsilon^{1 / 2} \rightarrow 0$ as $\varepsilon \rightarrow 0$, but this is not proved). In any case, the proof of Theorem 1 given here is simpler than that of Skubenko; indeed, our proof is along the lines of the original proof of the case $n=3$ [3, pp. 78-79], but with one additional idea.

2. Proof of Theorem 1. We first require a generalization of Lemma 1 of [3].

LEMMA 1. Fix $m \geq 2$. Let $A=\left[a_{i j}\right]$ be an $m$ by $m$ matrix of real numbers with $\operatorname{det} A \neq 0$. Suppose not all of the numbers $a_{1 j} a_{11}^{-1}(j=2,3, \ldots, m)$ are rational. Then given any' $\tau>0$ there exists a $\sigma$, depending on $\tau$ and on the $a_{i j}$, such that for any $\lambda$ there are integers $u_{1}, \ldots, u_{m}$ such that

$$
\left|u_{1} a_{11}+u_{2} a_{12}+\cdots+u_{m} a_{1 m}-\lambda\right|<\tau
$$

and

$$
\left|u_{1} a_{i 1}+u_{2} a_{i 2}+\cdots+u_{m} a_{i m}\right| \leq \sigma \quad \text { for } i=2,3, \ldots, m .
$$

ProOF. This is an easy consequence of Kronecker's theorem on Diophantine approximation.

In order to apply Lemma 1 in the proof of Theorem 1, we shall need the following lemma about units in totally real fields. If $\alpha$ is a number in an algebraic number field of degree $d$, we let $\alpha=\alpha^{(1)}, \alpha^{(2)}, \ldots, \alpha^{(d)}$ denote the conjugates of $\alpha$. The elegant proof of Lemma 2, due to Swinnerton-Dyer, replaces a clumsier one of the author.

LEMMA 2. Suppose $K$ is a totally real algebraic number field of degree $r \geq 3$ and $\chi_{1}, \ldots, \chi_{r-1}$ are any multiplicatively independent units in $K$. Then for any $i \neq j, 1 \leq i, j \leq r$, such that $\left|\chi_{1}^{(i)}\right| \neq\left|\chi_{1}^{(j)}\right|$, the $r-2$ numbers

$$
\frac{\log \left|\chi_{k}^{(i)} / \chi_{k}^{(j)}\right|}{\log \left|\chi_{1}^{(i)} / \chi_{1}^{(j)}\right|} \quad(k=2,3, \ldots, r-1)
$$

are not all rational.

PROOF. We suppose that for some fixed $i, j$ and rational integers $p(k), q(k) \neq 0$ we have

$$
\frac{\log \left|\chi_{k}^{(i)} / \chi_{k}^{(j)}\right|}{\log \left|\chi_{1}^{(i)} / \chi_{1}^{(j)}\right|}=\frac{p(k)}{q(k)} \quad(k=2,3, \ldots, r-1)
$$

we shall deduce a contradiction. We start with some simplifications, none of which alter the multiplicative independence of the $\chi_{k}$. First we square each $\chi_{k}$; then (1) still holds and we can remove the absolute value signs in (1) since every $\chi_{k}$ is totally positive. Now we define units

$$
y_{k}=\chi_{k}^{q(k)} \chi_{1}^{-p(k)} \quad(k=2,3, \ldots, r-1) ;
$$

since $q(k) \neq 0$, the $y_{k}(k=2,3, \ldots, r-1)$ are multiplicatively independent. Now (1) gives

$$
\log \left(y_{k}^{(i)} / y_{k}^{(j)}\right)=q(k) \log \left(\chi_{k}^{(i)} / \chi_{k}^{(j)}\right)-p(k) \log \left(\chi_{1}^{(i)} / \chi_{1}^{(j)}\right)=0,
$$

whence $y_{k}^{(i)}=y_{k}^{(j)}$ since $y_{k}$ is totally positive. 
Now let $L$ be the subfield of $K$ made up of those $b$ such that $b^{(i)}=b^{(j)}$; since $L$ is a proper subfield of $K$, it has degree at most $\frac{1}{2} r$. But (2) gives $r-2$ multiplicatively independent units of $L$, so we obtain

$$
r-2 \leq \frac{1}{2} r-1
$$

by Dirichlet's unit theorem. This contradiction proves Lemma 2.

As we saw in the Introduction, the form $f$ in Theorem 1 is essentially the product of $n$ linear forms whose coefficients lie in some totally real algebraic number field $K$ of degree $n$. In order to simplify the notation, we take $n=4$ for the rest of this section. Our arguments in the rest of the section clearly apply for all $n \geq 4$, and the case $n=3$ is easier.

Thus we may suppose

$$
f=L_{1} L_{2} L_{3} L_{4}
$$

where the $L_{i}$ are conjugate linear forms in $x_{1}, x_{2}, x_{3}, x_{4}$ with coefficients in the conjugate fields of $K$. If we define

$$
L_{i}^{*}=\sum_{j=1}^{4} \varepsilon_{i j} L_{j} \quad\left(1 \leq i \leq 4, \varepsilon_{j j}=1 \text { for } 1 \leq j \leq 4\right)
$$

and

$$
f^{*}=\left(1+\varepsilon_{0}\right) L_{1}^{*} L_{2}^{*} L_{3}^{*} L_{4}^{*}
$$

then the forms $f^{*}$ make up a neighborhood of $f$ (in the sense defined in the Introduction) if we put bounds on $\varepsilon_{0}$ and the twelve $\varepsilon_{i j}(i \neq j)$. Note $f^{*}$ is a multiple of $f$ if and only if the $\varepsilon_{i j}, i \neq j$, are all zero.

We may suppose the conjugate fields of $K$ are ordered so that if $L_{1}\left(x_{1}, x_{2}, x_{3}, x_{4}\right)$ $=\xi$, then

$$
L_{j}\left(x_{1}, x_{2}, x_{3}, x_{4}\right)=\xi^{(j)} \quad(1 \leq j \leq 4) .
$$

The set of all values taken on by $L_{1}$ for integral $x_{i}$ is a module, and we fix three positive independent units $\chi_{i}(1 \leq i \leq 3)$ in the coefficient ring of this module such that $\chi_{i}^{(j)}>0$ for $1 \leq i \leq 3$ and $1 \leq j \leq 4$. If $\mu=\chi_{1}^{p} \chi_{2}^{q} \chi_{3}^{r}$, this implies that for suitable integers $y_{i}$ we have

$$
L_{j}\left(u_{1}, y_{2}, y_{3}, y_{4}\right)=\mu^{(j)} \xi^{(j)} \quad(1 \leq j \leq 4) .
$$

We now prove a generalization of Lemma 2 of $[3]$.

LEMMA 3. Given any $\omega>0$ there exists a $C$, depending on $\omega$ and the units $\chi_{i}$, with the following property: If $\Psi$ is given, $0<\Psi<1$, then there exist integers $p, q, r$, depending on $\omega$ and $\Psi$, such that the unit $\theta=\chi_{1}^{p} \chi_{2}^{q} \chi_{3}^{r}$ satisfies

$$
\omega \theta<\left|\theta-\Psi \theta^{(2)}\right|<2 \omega \theta
$$

and

$$
\Psi^{1 / 2} \theta^{(i)}<C \theta^{(j)} \quad(1 \leq i, j \leq 4,(i, j) \neq(2,1)) .
$$

ProOF. We apply Lemma 1 with $m=3$ and

$$
a_{1 j}=\log \left(\chi_{j} / \chi_{j}^{(2)}\right), \quad a_{2 j}=\log \chi_{j} \chi_{j}^{(2)}, \quad a_{3 j}=\log \chi_{j} \chi_{j}^{(2)} \chi_{j}^{(3)} \quad(1 \leq j \leq 3) .
$$


The matrix $A=\left[a_{i j}\right]$ is clearly a nonzero multiple of the regulator matrix of $F$, so $\operatorname{det} A \neq 0$. Also, by Lemma 2 with $r=4$, the numbers $a_{12} a_{11}^{-1}$ and $a_{13} a_{11}^{-1}$ are not both rational, so the hypotheses of Lemma 1 are satisfied. (We can assume $a_{11} \neq 0$ by renumbering conjugates.) We choose

$$
\lambda=\log \Psi, \quad \tau=\log (1+\omega)
$$

in Lemma 1 and we may suppose $\omega<1$. We take $p=u_{1}, q=u_{2}, r=u_{3}$, where $u_{1}, u_{2}, u_{3}$ are the integers obtained in Lemma 1 ; then Lemma 1 gives

$$
1-\omega<(1+\omega)^{-1}<\Psi \theta^{(2)} \theta^{-1}<1+\omega
$$

and

$$
c^{-1} \leq \theta \theta^{(2)} \leq c, \quad c^{-1} \leq \theta \theta^{(2)} \theta^{(3)} \leq c,
$$

where $c=e^{\sigma}$ depends only on $\omega$ and the $\chi_{i}$.

It follows immediately from (5) that

$$
\left|\theta-\Psi \theta^{(2)}\right|<\omega \theta
$$

and (5) and (6) together imply

$$
\begin{gathered}
\left(c^{-1}(1+\omega)^{-1} \Psi\right)^{1 / 2}<\theta<(c(1+\omega) \Psi)^{1 / 2} \\
\left(c^{-1}(1+\omega)^{-1} \Psi^{-1}\right)^{1 / 2}<\theta^{(2)}<\left(c(1+\omega) \Psi^{-1}\right)^{1 / 2} \\
c^{-2} \leq \theta^{(3)} \leq c^{2}, \quad c^{-1} \leq \theta^{(4)} \leq c
\end{gathered}
$$

since $\theta \theta^{(2)} \theta^{(3)} \theta^{(4)}=1$. Since $c$ is independent of $\Psi,(8),(9)$, and (10) imply that (4) holds for a constant $C$ depending only on $\omega$ and the $\chi_{i}$.

We may suppose $\omega<2 / 3$, and then we may replace $\Psi$ by $2 \Psi /(2-3 \omega)$ and $\omega$ by $\omega /(2-3 \omega)$ in the lemma. Now (7) implies (3) and we can change the value of $C$ so that (4) still holds. This completes the proof of Lemma 3.

We can now prove Theorem 1. It is enough to show that given any $\delta>0$, the inequality

$$
0<\left|f^{*}\right|=\left|L_{1}^{*} L_{2}^{*} L_{3}^{*} L_{4}^{*}\right|<\delta
$$

is solvable for any form $f^{*}$, not a multiple of $f$, in some neighborhood of $f$ : for if $f^{*}$ takes on some value $\delta_{0}$, then it also takes on all values $m^{4} \delta_{0}$ for integer $m$, and we need only choose $\delta_{0}$ so small that

$$
\delta_{1}<m^{4} \delta_{0}<(m+1)^{4} \delta_{0}<\delta_{2}
$$

for some integer $m$.

Plainly we may suppose $\varepsilon_{0}=0$ without loss of generality, and we shall also assume

$$
\varepsilon_{12}=\max _{i \neq j}\left|\varepsilon_{i j}\right|>0
$$

this is one of 24 possible cases for the maximum of $\varepsilon_{i j}(i \neq j)$, sign being taken into account, and all of these cases can be handled in the same way.

In order to satisfy (11) we shall take

$$
L_{j}=\left(\chi_{1}^{p} \chi_{2}^{q} \chi_{3}^{r}\right)^{(j)} \xi^{(j)} \quad(1 \leq j \leq 4)
$$


where $\xi$ is a fixed value of $L_{1}$ and $p, q, r$ are chosen so that $L_{1}^{*}$ is small compared to $L_{1}$, but $L_{j}^{*}$ is similar in size to $L_{j}$ for $j=2,3,4$. We fix $\xi$ so that $\xi \xi^{(2)}<0$ and define

$$
\Psi=-\varepsilon_{12} \xi^{-1} \xi^{(2)}>0 .
$$

Now we have $\Psi \rightarrow 0$ as $\varepsilon_{12} \rightarrow 0$.

We choose a small $\omega$ and take $\varepsilon_{12}$ so small that $\Psi<1$, so Lemma 3 applies and we define the $L_{j}$ by (12), where $p, q, r$ are the integers determined in Lemma 3. Now (3) gives

$$
\omega \theta|\xi|<\left|\theta \xi+\varepsilon_{12} \theta^{(2)} \xi^{(2)}\right|<2 \omega \theta|\xi|,
$$

so (4) and (13) imply

$$
\begin{aligned}
\left|L_{1}^{*}\right| & \geq\left|\theta \xi+\varepsilon_{12} \theta^{(2)} \xi^{(2)}\right|-\left|\varepsilon_{13} \theta^{(3)} \xi^{(3)}\right|-\left|\varepsilon_{14} \theta^{(4)} \xi^{(4)}\right| \\
& >\omega \theta|\xi|-C\left|\xi \xi^{(3)} / \xi^{(2)}\right| \Psi^{1 / 2} \theta-C\left|\xi \xi^{(4)} / \xi^{(2)}\right| \Psi^{1 / 2} \theta>0
\end{aligned}
$$

provided $\varepsilon_{12}$ (and so $\Psi$ ) is small enough. Also (3), (4), and (13) give

$$
\begin{aligned}
\left|L_{1}^{*}\right| & <\left|\theta \xi+\varepsilon_{12} \theta^{(2)} \xi^{(2)}\right|+\left|\varepsilon_{13} \theta^{(3)} \xi^{(3)}\right|+\left|\varepsilon_{14} \theta^{(4)} \xi^{(4)}\right| \\
& <2 \omega \theta|\xi|+\varepsilon_{12}^{1 / 2}\left|\xi^{-1} \xi^{(2)}\right|^{-1 / 2}<3 \omega \theta|\xi|
\end{aligned}
$$

if $\varepsilon_{12}$ is small enough. By a similar but simpler argument

$$
0<\left|L_{j}^{*}\right|<2 \theta^{(j)}\left|\xi^{(j)}\right| \quad(j=2,3,4)
$$

if $\varepsilon_{12}$ is small enough; so we finally obtain

$$
0<\left|L_{1}^{*} L_{2}^{*} L_{3}^{*} L_{4}^{*}\right|<24 \omega\left|\xi \xi^{(2)} \xi^{(3)} \xi^{(4)}\right| .
$$

Since we may choose $\omega$ as small as we wish, the proves (11) and so Theorem 1.

\section{REFERENCES}

1. J. W. S. Cassels, An introduction to diophantine approximation, Cambridge Univ. Press, 1957.

2. _ An introduction to the geometry of numbers, Springer-Verlag, Berlin, 1959.

3. J. W. S. Cassels and H. P. F. Swinnerton-Dyer, On the product of three homogeneous linear forms and indefinite ternary quadratic forms, Philos. Trans. Roy. Soc. London Ser A 248 (1955), 73-96.

4. B. F. Skubenko, On the product of $n$ linear forms in $n$ variables, Trudy Mat. Inst. Steklov. 158 (1981), 175-179. (Russian)

5. I Isolation theorems for decomposable forms over totally real algebraic number fields of degree $n \geq 3$, Zap. Nauchn. Sem. Leningrad. Otdel. Mat. Int. Steklov. (LOMI) 112 (1981), 167171. (Russian)

Department of Mathematics, State University of NeW York, Buffalo, NeW YORK 14214 